\title{
Synthesis of Bare Iron Nanoparticles from Ferrocene Hexane Solution by Femtosecond Laser Pulses
}

\section{Takuya Okamoto, Takahiro Nakamura, Ryo Kihara, Tsuyoshi Asahi, Kenji Sakota, Tomoyuki Yatsuhashi}

\begin{tabular}{|c|c|}
\hline Citation & ChemPhysChem, 19(19); 2480-2485 \\
\hline Issue Date & 2018-10-05 \\
\hline Type & Journal Article \\
\hline Textversion & Author \\
\hline Rights & $\begin{array}{l}\text { This is the peer-reviewed version of the following article: ChemPhysChem. Vol.19, } \\
\text { Issue 19, pp.2480-2485, which has been published in final form at } \\
\text { https://doi.org/10.1002/cphc. } 201800436 \text {. This article may be used for } \\
\text { non-commercial purposes in accordance with Wiley-VCH Terms and Conditions for } \\
\text { Self-Archiving. }\end{array}$ \\
\hline DOI & 10.1002/cphc.201800436 \\
\hline
\end{tabular}

\author{
Self-Archiving by Author(s) \\ Placed on: Osaka City University
}

OKAMOTO, T., NAKAMURA, T., KIHARA, R., ASAHI, T., SAKOTA, K., \& YATSUHASHI, T. (2018). Synthesis of Bare Iron Nanoparticles from Ferrocene Hexane Solution by Femtosecond Laser Pulses. ChemPhysChem. 19, 2480-2485. Doi:10.1002/cphc.201800436 


\title{
Synthesis of Bare Iron Nanoparticles from Ferrocene Hexane Solution by Femtosecond Laser Pulses
}

\author{
Takuya Okamoto, ${ }^{[a]}$ Takahiro Nakamura, ${ }^{[b]}$ Ryo Kihara, ${ }^{[c]}$ Tsuyoshi Asahi, ${ }^{[c]}$ Kenji Sakota, ${ }^{\left[{ }^{[a]}\right.}$ Tomoyuki \\ Yatsuhashi*[a]
}

\begin{abstract}
Iron-based nanoparticles (FeNPs) have unique and attractive properties such as superparamagnetism, biocompatibility and catalytic activity. Although the synthesis of precious metal NPs from a metal in liquid and/or metal salt solution by a pulsed laser has been well reported, little has been examined about the production of FeNPs. Here we report the synthesis of spherical NPs of iron oxide (magnetite) without wearing carbon shells from ferrocene hexane solution by femtosecond NIR laser pulses. Nanosecond UV laser is also used to compare the time evolutions of the particle size distribution. The size of NPs remains constant even by the long-term femtosecond laser irradiation, whereas it grows with nanosecond laser pulses. The primary particles are generated by photochemical reactions regardless of pulse durations; however, the fragmentation of NPs by the successive femtosecond laser pulses regulates the particle size.
\end{abstract}

\section{Introduction}

Iron-based nanoparticles (FeNPs) showing superparamagnetism ${ }^{[1]}$ and biocompatibility ${ }^{[2]}$ have potential applications such as data storage, ${ }^{[3]}$ ferrofluids, ${ }^{[4]}$ biosensor, ${ }^{[5]}$ bioimaging, ${ }^{[6]}$ and drug delivery. ${ }^{[7]}$ Furthermore, they have been used in olefin formation, ${ }^{[8]}$ photooxidation, ${ }^{[9]}$ and oxygen reduction reaction ${ }^{[10]}$ as sustainable catalysts. FeNPs have been synthesized by chemical reduction, ${ }^{[11]}$ pyrolysis, ${ }^{[12]}$ and solvothermolysis. ${ }^{[13]}$ These chemical synthesis methods sometimes require severe reaction conditions and/or additive reagents such as reductants and surfactants. In contrast, the synthesis of NPs without the aid of additive reagents has been proposed in gas, solid, and liquid phase by using a pulsed laser. Iron (bcc) core surrounded by carbon shell are formed from ferrocene $\left(\mathrm{FeCp}_{2}\right)$ vapor by using a nanosecond VUV laser (193 $\mathrm{nm}, 15 \mathrm{~ns}) \cdot{ }^{[14]}$ Amorphous iron core with carbon onion shells are synthesized from $\mathrm{FeCp}_{2}$ pellets by using a nanosecond visible

[a] T. Okamoto, Dr. K. Sakota, Prof. Dr. T. Yatsuhashi

Graduate School of Science

Osaka City University

3-3-138 Sugimoto, Sumiyoshi-ku, Osaka 558-8585 (Japan)

E-mail: tomo@sci.osaka-cu.ac.jp

[b] Dr. Takahiro Nakamura

Institute of Multidisciplinary Research for Advanced Materials

Tohoku University

2-1-1 Katahira, Aoba-ku, Sendai 980-857 (Japan)

[c] Ryo Kihara, Prof. Dr. Tsuyoshi Asahi

Graduate School of Science and Engineering

Ehime University

3 Bunkyo-cho, Matsuyama 790-8577 (Japan)

Supporting information for this article is given via a link at the end of the document. laser $(532 \mathrm{~nm}, 5 \mathrm{~ns}) \cdot{ }^{[15]}$ Under a supercritical condition, iron (bcc or fcc) core covered with carbon shells are produced from $\mathrm{FeCp}_{2}$ (benzene, 266 nm, $4.3 \mathrm{~ns} ;{ }^{[16]} \mathrm{CO}_{2}, 193 \mathrm{~nm}, 30 \mathrm{~ns} ;{ }^{[17]} 266 \mathrm{~nm}, 4.3$ $\left.\mathrm{ns}^{[17]}\right)$.

Practically, the synthesis of NPs in liquid phase has advantages because of widely controllable experimental conditions and reactant variations. The reactant is prepared as a solid material immersed in inert liquid or a solute in solvent. The former method is known as laser ablation in liquid $(L A L) \bar{r}^{[18-25]}$, in which the ablated hot materials are rapidly cooled and aggregated to form NPs. In the latter case, namely laser reaction in liquid ( $L R L)$, bare metals prepared by photochemical reaction of reactant finally form NPs. It is emphasized that metal NPs are formed by the reduction of metal ions by solvated electrons when femtosecond laser pulses are utilized for LRL, because the density of electron generated by the ionization of solvent reaches to $10^{18} \mathrm{~cm}^{-3} \cdot{ }^{[26]} \mathrm{A}$ variety of precious metal $\mathrm{NPs}^{[27-29]}$ as well as multimetallic alloy NPs have been prepared from aqueous metal salts. ${ }^{[30-33]}$ Nakashima et al. have investigated the mechanism of metal ion reduction ${ }^{[34-36]}$ and successive NPs

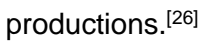

Various reports have been published on the production of FeNPs by LAL. ${ }^{[37-43]} \mathrm{Hu}$ et al. have reported the formation of $\mathrm{Fe}_{2} \mathrm{O}_{3}$ core coated by diamond-like carbon by the $\mathrm{LAL}$ of an iron plate in hexane $(800 \mathrm{~nm}, 90 \mathrm{fs}) .{ }^{[37]}$ Amendola et al. have reported that various FeNPs such as iron carbide, iron oxide and iron with carbon shells are formed from an iron plate by varying the organic solvent $(1064 \mathrm{~nm}, 9 \mathrm{~ns}) .{ }^{[38]}$ As for LRL, iron core covered with carbon shells were produced from $\mathrm{FeCp}_{2}$ solution under different conditions (xylene, $355 \mathrm{~nm}, 5 \mathrm{~ns} ;{ }^{[44]}$ hexane, 193 $\left.\mathrm{nm}^{[45]}\right)$. However, these LRL studies have focused attention not on the particle size but mainly on the composition of NPs. Moreover, LRL of organoiron complex solution by using femtosecond laser pulses has rarely been examined. Wesolowski et al. have carried out $L R L$ of $\mathrm{FeCp}_{2}$ benzene solution by femtosecond NIR laser pulses ( $800 \mathrm{~nm}, 100 \mathrm{fs})$, but they investigate that the dominant product is thin carbon microshells (4-25 $\mu \mathrm{m}$ diameter, 30-60 nm thickness). ${ }^{[66]}$

In this study, we report the synthesis of spherical iron oxide (magnetite/wüstite) and iron (bcc) NPs without carbon shells from $\mathrm{FeCp}_{2}$ hexane solution by femtosecond NIR laser pulses $(0.8 \mu \mathrm{m}, 40 \mathrm{fs})$. The size distributions, elemental mappings and structural information of these FeNPs were investigated. Nanosecond UV laser pulses ( $355 \mathrm{~nm}, 8 \mathrm{~ns}$ ) were also used for comparison purpose. The regulation of particle size even with prolonged femtosecond laser irradiation is discussed based on the time evolution of the size distribution of NPs on laser irradiation time.

\section{Results}




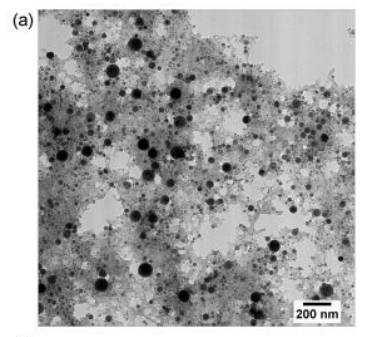

(c)

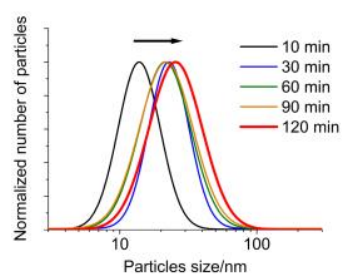

(b)

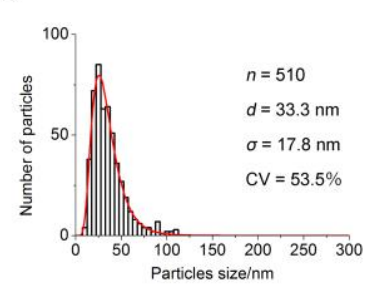

(d)

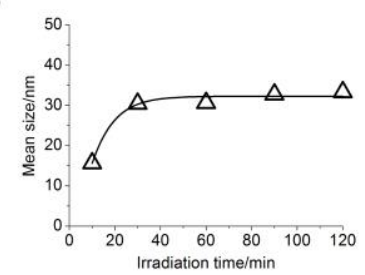

Figure $1 \mathrm{a})$ TEM image (scale bar: $200 \mathrm{~nm}$ ) and b) size distribution after the 120-min femtosecond NIR laser irradiation. The solid line in b) is a fitting curve by a log-normal distribution function. $n, d, \sigma$, and CV denote the number of particles, mean size, standard deviation, and the coefficient of variation, respectively. The time evolution of $c$ ) the normalized size distributions fitted by log-normal distribution functions and d) the mean size of the spherical nanoparticles. The number of counted particles was ca. 500 for each data. Line in $\mathrm{d})$ is drawn to guide the eye. $\mathrm{FeCp}_{2}$ in hexane $\left(1.0 \times 10^{-2} \mathrm{~mol} \mathrm{dm}^{-3}\right)$ was used.

Transmission electron microscope (TEM) images showed that high-contrast spherical NPs surrounded by low-contrast agglomerates were produced from $\mathrm{FeCp}_{2}$ hexane solution $\left(1.0 \times 10^{-2} \mathrm{~mol} \mathrm{dm}^{-3}\right)$ by femtosecond NIR laser pulses (Figure 1a). It should be noted that carbon micro-shells ${ }^{[46]}$ were not found by the TEM observations. The size distribution was obtained by counting the spherical NPs appeared in Figure 1a (Figure 1b). The distribution of NP size was well fitted by a lognormal distribution function. The mean size $(d)$ of the spherical NPs obtained by the 120 -min laser irradiation was $33.3 \pm 17.8 \mathrm{~nm}$ The coefficient of variation (CV), which equals to the standard deviation $(\sigma)$ divided by mean size, was $53.5 \%$. Figures $1 \mathrm{c}$ and $1 d$ show the time evolution of the normalized log-normal distribution and the mean size of NPs. The peak of size distribution and mean size increased from 10 to $30 \mathrm{~min}$, and stayed constant up to $120 \mathrm{~min}$.

Nanosecond UV laser pulses were also used to synthesize NPs for comparison purpose. Figure 2 shows the results obtained as in the cases of femtosecond NIR laser irradiation experiments. The mean size and CV of spherical NPs obtained by the 120 -min laser irradiation were $47.2 \pm 27.7 \mathrm{~nm}$ and $58.7 \%$, respectively. It should be mentioned that the time evolution of log-normal distribution and mean size showed step like behaviour. This step like feature was confirmed by the experiments carried out at three different days.

Although the experimental conditions (wavelength, pulse durations, pulse energy, number of laser shots, etc.) are virtually different, the mean size of NPs remained constant by prolonged laser irradiation in the case of femtosecond NIR laser experiments. Therefore, we further investigated the structure and elemental composition of NPs obtained by using femtosecond NIR laser pulses.

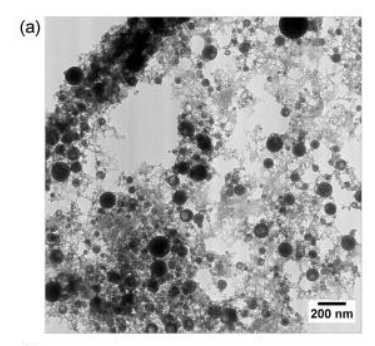

(c)
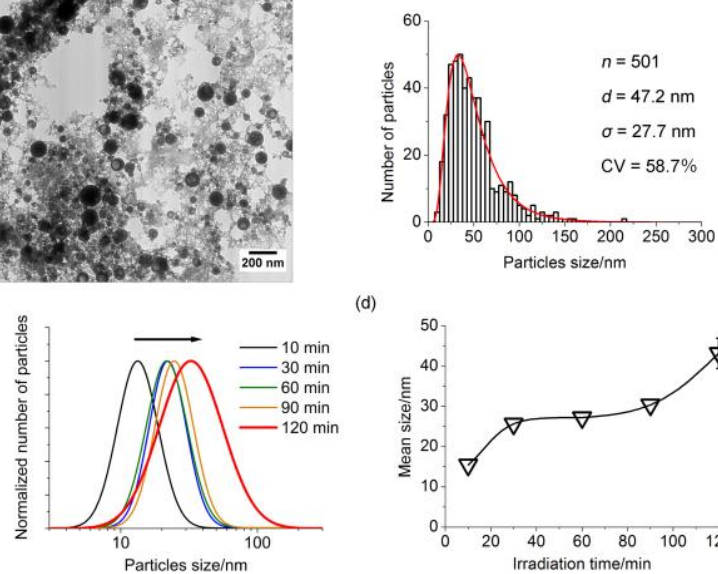

(d)

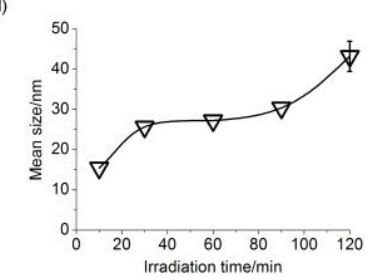

Figure 2 a) TEM image (scale bar: $200 \mathrm{~nm}$ ) and b) size distribution after the 120-min nanosecond UV laser irradiation. The solid line in b) is a fitting curve by a log-normal distribution function. $n, d, \sigma$, and $C V$ denote the number of particles, mean size, standard deviation, and the coefficient of variation, respectively. The time evolution of $\mathrm{c}$ ) the normalized size distributions fitted by log-normal distribution functions and d) the mean size of the spherical nanoparticles. The vertical bars in d) give the standard deviation obtained in the three measurements. The number of counted particles was ca. 500 for each data. Line in d) is drawn to guide the eye. $\mathrm{FeCp}_{2}$ in hexane $\left(1.0 \times 10^{-2} \mathrm{~mol}\right.$ $\mathrm{dm}^{-3}$ ) was used.

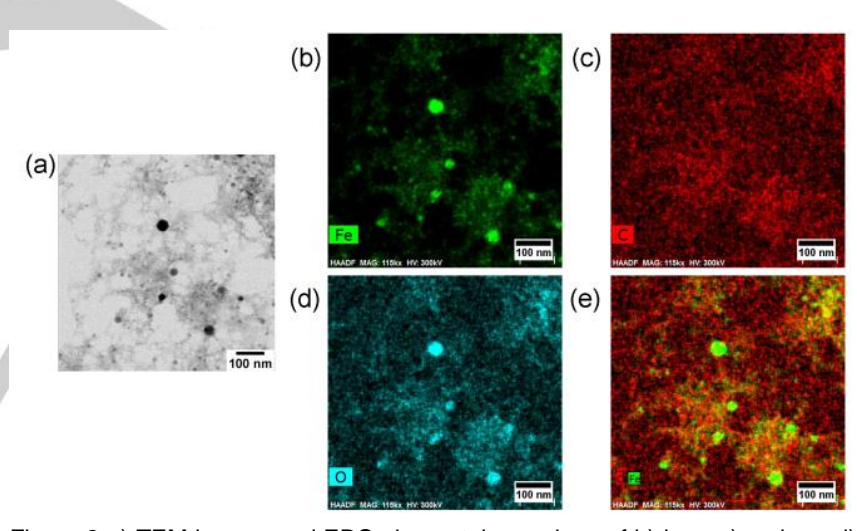

Figure 3 a) TEM image, and EDS elemental mappings of b) iron, c) carbon, d) oxygen, and e) reconstructed image of iron and carbon of nanoparticles synthesized from $\mathrm{FeCp}_{2}$ hexane solution $\left(1.0 \times 10^{-3} \mathrm{~mol} \mathrm{dm}^{-3}\right)$ by the $25-\mathrm{min}$ femtosecond laser irradiation. Scale bar $100 \mathrm{~nm}$. The corresponding TEM image and size distribution are shown in Figure $\mathrm{S} 1$.

The elemental mappings using TEM equipped with an energy dispersive X-ray spectrometer (TEM-EDS) were performed for the NPs obtained by the 25-min femtosecond laser irradiation. TEM image and size distribution are shown in Figure S1. Figure 3 shows TEM image and EDS elemental mappings of iron, carbon, oxygen, and a reconstructed image of iron and carbon. Although the spatial resolution of TEM-EDS is limited, we can recognize that the distributions of iron and oxygen are well coincided with that of the spherical NPs larger than ca. $10 \mathrm{~nm}$ in diameter. Therefore, most of the NPs are assigned to be oxidized iron particles. The low-intensity part of signals seems to be overlapped. However, this artefactual overlap may be attributed to the common background signal of 
$\mathrm{Ka}$ (carbon, oxygen) and La (iron) lines used for EDS measurements. From these observations, it is safe to say that the spherical iron oxide NPs formed by femtosecond laser irradiation of $\mathrm{FeCp}_{2}$ hexane solution do not wear carbon shells but are only loosely surrounded by carbon agglomerates.

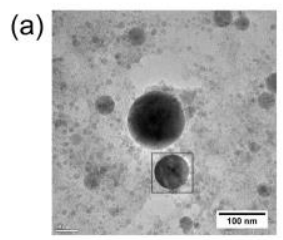

(d)

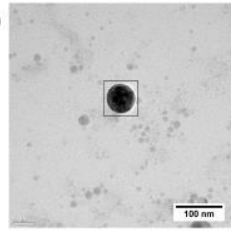

(b)

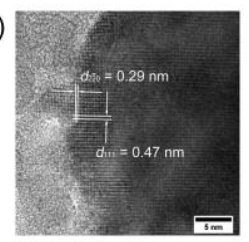

(e)

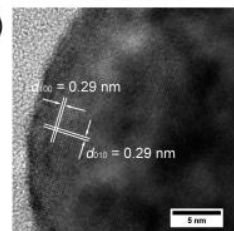

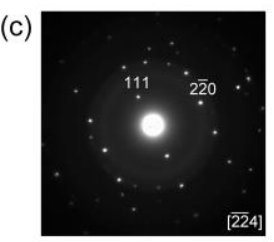

(f)

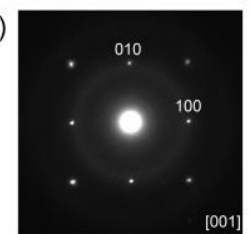

Figure 4 a) and d) TEM images (scale bar: $100 \mathrm{~nm}$ ), b) and e) HR-TEM images (scale bar: $5 \mathrm{~nm}$ ), and c) and f) SAED patterns of nanoparticles synthesized from $\mathrm{FeCp}_{2}$ hexane solution $\left(1.0 \times 10^{-3} \mathrm{~mol} \mathrm{dm}^{-3}\right)$ by the 25 -min femtosecond laser irradiation. Values in square brackets denote the zone axis of electron beam incidence.

A NP indicated by a black square in Figure 4 a was analyzed by high-resolution TEM (HR-TEM, Figure $4 b)$ and selected area electron diffraction (SAED, Figure 4c) measurements. The interplanar spacings $(0.29$ and $0.47 \mathrm{~nm})$ of this spherical NP observed by HR-TEM were well coincided with those calculated for the corresponding crystal planes (111) and (2 $\overline{2} 0)$ of magnetite $\left(\mathrm{Fe}_{3} \mathrm{O}_{4}\right.$, the unit length: $\left.8.3941 \AA^{[47]}\right)$. X-ray diffraction (XRD) patterns of FeNPs collected after the 120-min laser irradiation (Figure S2) showed the diffraction peaks of magnetite. These observations lead us to assign the dominant FeNP to be magnetite. We also observed the XRD patterns of wüstite ( $\mathrm{FeO}$, Figure S2). Moreover, we found the FeNP of different structure. The interplanar spacings ( 0.29 and $0.29 \mathrm{~nm}$, Figure $4 \mathrm{e})$, and the crystal planes (010) and (100) shown in Figure $4 f$ of the NP indicated by a black square in Figure $4 \mathrm{~d}$ were in accordance with that of iron (bcc) structure (the unit length: $0.2874 \mathrm{~nm}^{[48]}$ ).

\section{Discussion}

Spherical and bare FeNPs were formed from $\mathrm{FeCp}_{2}$ hexane solution both by femtosecond NIR and nanosecond UV laser pulses in this study. It should be mentioned that the results of the similar femtosecond laser irradiation experiments previously carried out by Wesolowski et al. are entirely different. They have reported that carbon micro-shells (4-25 $\mu \mathrm{m}$ diameter, 30-60 nm thickness) are dominantly formed from relatively high concentration of $\mathrm{FeCp}_{2}\left(0.75 \mathrm{~mol} \mathrm{dm}^{-3}\right)$ benzene solution by femtosecond NIR laser pulses $(800 \mathrm{~nm}, 100 \mathrm{fs}, 300 \mu \mathrm{J}) .{ }^{[46]}$ Although the role of $\mathrm{FeCp}_{2}$ in carbon micro-shell production is not certain, the origin of carbon micro-shell might be the solvents used in their study. Our previous works have demonstrated that carbon materials (NPs, diamond-like carbons) and their agglomerate are not formed from aliphatic hydrocarbons ${ }^{[49]}$ but from aromatic hydrocarbons by femtosecond laser pulses. ${ }^{[49-52]}$ Aromatic solvent such as benzene and toluene may partially decompose to fragments that can form carbon nanoparticles. In contrast, aliphatic solvent such as hexane is expected to be decomposed into small fragments that form polyynes. ${ }^{[53]}$

The present experiments investigated that the resultant FeNPs were not covered with carbon shells both in the cases of femtosecond NIR and nanosecond UV laser irradiations. On the contrary, Park et al. have shown that iron (bcc) cores formed from $\mathrm{FeCp}_{2}$ xylene solution by nanosecond UV laser pulses (355 $\mathrm{nm}, 5 \mathrm{~ns})$ are covered with graphitic carbon shells. ${ }^{[44]}$ We conclude that the fragments formed from aromatic solvent would form carbon layers on a particle core, while the small fragments formed form aliphatic solvent would not easily gather to form carbon shells. It is also suggested that the carbon agglomerates found in this study are originated in cyclopentadienyl ligands.

There are numbers of unclarified points in FeNPs production from organometallic solution by LRL. What factors do determine the composition of FeNPs? How small FeNPs are formed? The present study carried out under air atmosphere showed that $\mathrm{Fe}$ (bcc) in addition to $\mathrm{Fe}_{3} \mathrm{O}_{4}$ was observed at relatively short irradiation time (25 $\mathrm{min})$. However, the corresponding diffraction peak of iron (bcc) $\left(2 \theta=45^{\circ}\right)$ was not observed in the XRD measurement of FeNPs obtained by the 120-min laser irradiation. This result indicates that iron oxides are the main products after the prolonged laser irradiation. The oxidation of iron core may occur by the exposure to air during the laser irradiation as well as the treatment before TEM measurements. In addition, highly reactive oxidant such as hydroxyl radical is formed by the femtosecond laser irradiation to water ${ }^{[54]}$ that is contaminated in hexane. It is proposed to carry out experiments in anhydrous solvent and under inert atmosphere to obtain iron (bcc) core.

In this study, we judge that it would be more advantageous to use a femtosecond rather than nanosecond laser to produce smaller FeNPs under the continuous laser irradiation condition. The growth of particle by nanosecond laser pulses is known as laser melting in liquid (LML). ${ }^{[18,55,56]}$ Ishikawa et al. have reported that the diameter of iron oxide NPs increase from $250 \mathrm{~nm}$ (reactant) to $400-500 \mathrm{~nm}$ (product) by LML (532 nm, $5 \mathrm{~ns}$ ). ${ }^{[57]}$ Initial NPs are exposed to low peak laser power but $10^{6}$ times longer duration than that of femtosecond laser pulses. Therefore, the multiphoton ionization of NPs is not expected but the particles are melted and merged. It is reasonable to conclude that the increase of the diameter of FeNPs is promoted by LML in the present nanosecond UV laser irradiation experiments. The step-like feature observed in the time evolution of particle size (Figure $2 \mathrm{~d}$ ) is explained in terms of the induction period before reaching the critical concentration of NPs to be merged.

In contrast to LML, the size reduction of NPs by laser pulses, namely laser fragmentation in liquid (LFL), has been proposed. ${ }^{[18,58]}$ The thermal and/or electrostatic process has been recognized, and controversy as to arguments regarding the fragmentation mechanism is ongoing. ${ }^{[59-62]}$ One of the candidate is Coulomb explosion, which is destructive dissociations due to the strong Coulomb repulsion of positive charges. Coulomb explosion of multiply charged molecules has been widely studied in the gas phase. ${ }^{[63.64]}$ Moreover, recent 
studies have presented the examples of Coulomb explosion of molecules in liquid helium droplets ${ }^{[65]}$ as well as alkali metals in water. ${ }^{[66]}$ Briefly, initially formed NPs are ionized by succeeding femtosecond laser pulses followed by the fragment production due to Coulomb repulsion in charged NPs. We conclude that the equilibrium between fragmentation of NPs and aggregation of fragments would be operative in the present femtosecond laser irradiation experiments. Consequently, the peak power of laser pulse is important factor governing the former process that limit the particle size without the aid of any capping reagents such as surfactants. However, the peak power of femtosecond laser pulse in liquid is hardly determined due to the group velocity dispersion. In addition, the intensity-clamping in femtosecond laser generated plasma filament may prevent us from varying the laser intensity in liquid. ${ }^{[67]}$

The mechanism of primary FeNPs formation process in LRL is also important to consider the effect of laser pulse durations on the particle growth process. The photochemical reactions of $\mathrm{FeCp}_{2}$ is operative when nanosecond UV laser pulses are used, while both photochemical reactions and ionization are expected to occur when femtosecond NIR laser is utilized. As for the productions of carbon NPs by femtosecond NIR laser pulses, the active species to form NPs is dependent on the reactants. For example, hydroxyl radicals act as oxidants when the benzene/water bilayer solution is used as a reactant, ${ }^{[49,50]}$ whereas solvated electrons act as reductants when hexafluorobenzene ${ }^{[51]}$ or dichloromethane/water bilayer solution is used as a reactant. ${ }^{[6]}$ The production of precious metal NPs from aqueous solution by femtosecond NIR laser pulses is explained by the reduction of metal ions by hydrated electrons. ${ }^{[26,34]}$ Solvated electrons may be formed by the ionization of $\mathrm{FeCp}_{2}$ and hexane in this study. However, the reduction of iron ions by solvated electrons may not be important for the formation of primary FeNPs in this study. The previous work has demonstrated that the reduction of $\mathrm{Fe}^{3+}$ to $\mathrm{Fe}^{2+}$ occurs when $\mathrm{K}_{3} \mathrm{Fe}\left(\mathrm{C}_{2} \mathrm{O}_{4}\right)_{3}$ aqueous solution is exposed to femtosecond laser pulses; ${ }^{[34]}$ however, further reduction of $\mathrm{Fe}^{2+}$ is not observed probably because this reaction is highly endothermic. We conclude that the photochemical reactions of $\mathrm{FeCp}_{2}$ forming bare iron and ligands would be a primary process both in nanosecond UV and femtosecond NIR laser experiments. The multiphoton absorption process should trigger the photochemical reaction in the latter case.

The synthesis of metal NPs from organometallic solution by $\mathrm{LRL}$ is attractive method due to the simplicity but has some difficulties for engineering applications. In addition to the low production yield, the problems to be solved are contamination of carbon agglomerates and the control of elemental composition. Moreover, the strategy to produce mono-dispersed smaller NPs $(<10 \mathrm{~nm})$ is expected. As we conclude that the main carbon source is cyclopentadienyl ligands, the amount of carbon could be reduced by replacing cyclopentadienyl ligands to readily degradable ligands. Oxidation number could be controlled by preparing the oxygen-free or oxygen-saturated condition. The repeated fragmentation and aggregation processes of NPs during femtosecond laser irradiation would determine the optimum particle size. Thus, the synthesis of mono-dispersed smaller NPs requires the optimization of laser irradiation conditions such as repetition rate as well as the reaction environments.

\section{Conclusion}

The bare iron nanoparticles without carbon shells were produced both by femtosecond NIR and nanosecond UV pulses when ferrocene hexane solution was used as a reactant. The factors that determine the bare as well as small particle production is the use of aliphatic solvent and femtosecond NIR rather than nanosecond UV laser pulses. Nanosecond VUV laser irradiation to $\mathrm{FeCp}_{2}$ dissolved in hexane ${ }^{[45]}$ has resulted in the formation of FeNPs covered with carbonaceous polymer due to the photochemical reactions of solute and solvent. In contrast, laser pulses used in this study are predominantly absorbed by solute to induce its photochemical reactions. As the carbon source should be ligands of reactants instead of solvent, we can reduce the amount of carbon agglomerate by choosing suitable ligands. As the particle size is determined by the balance between the fragmentation of NPs by successive femtosecond laser pulses and aggregation of fragments, we can control the size of NPs by altering this equilibrium. Femtosecond laser process can be one of the candidates to produce bare FeNPs without the use of any additive reagent, but further investigation about the effect of solvents, ligands and reaction environments, and laser irradiation conditions should be necessary to produce mono-dispersed single-nanometer size FeNPs.

\section{Experimental Section}

Ferrocene $\left(\mathrm{FeCp}_{2}\right.$, Aldrich, 98\%) and $n$-hexane (Nacalai Tesque, spectral grade, $\geq 96.0 \%$ ) were used without further purification. Femtosecond laser pulses $(0.8 \mu \mathrm{m}, 40 \mathrm{fs}, 0.4 \mathrm{~mJ}, 1 \mathrm{kHz})$ delivered from Ti:Sapphire laser (Thales Laser, Alpha 100/1000/XS hybrid) were focused on $\mathrm{FeCp}_{2}$ hexane solution in the quartz cuvette with a $1-\mathrm{cm}$ optical path length by using a plano-convex lens with a focal length of 50 $\mathrm{mm}$. Details of the laser experiments have been described elsewhere ${ }^{[49]}$. Nanosecond laser pulses ( $355 \mathrm{~nm}, 8 \mathrm{~ns}, 45 \mathrm{~mJ}, 10 \mathrm{~Hz}$ ) were focused on $\mathrm{FeCp}_{2}$ hexane solution in the quartz cuvette with a $4.5-\mathrm{cm}$ optical path length by using a plano-convex lens with a focal length of $30 \mathrm{~mm}$. Both femtosecond and nanosecond laser irradiation were performed under air atmosphere at room temperature (296 K).

The morphology and size distribution of the NPs were analyzed by using TEM (JEM-1010, JEOL) that was operated at an acceleration voltage of $80 \mathrm{kV}$. For the preparation of specimens for TEM observations, $10 \mu \mathrm{L}$ of sample solution was directly dropped onto a copper grid covered with amorphous carbon film (Nisshin EM Co., Ltd.) followed by drying in air atmosphere at room temperature. The mean size and size distribution of spherical particles were obtained first by marking NPs in TEM images and then by analyzing the binarized images (ex. Figure S1b) by using an image processing software (ImageJ $1.48 \mathrm{v}$ ) provided by National Institutes of Health. HR-TEM images and SAED patterns were obtained by using EM-002B (Topcon) operated at $200 \mathrm{kV}$. EDS mapping was performed by using Titan G2 Cubed (FEI) operated at $300 \mathrm{kV}$. In these TEM measurements (HR-TEM, SAED, EDS mapping), a copper grid covered with amorphous silicon film (Okenshoji) was used. The 
unreacted $\mathrm{FeCp}_{2}$ was sublimated in vacuum before TEM measurements due to its high vapor pressure $\left(0.774 \mathrm{~Pa}\right.$ at $\left.295.69 \mathrm{~K} \cdot{ }^{[69]}\right)$

\section{Acknowledgements}

The present research was supported in part by the Osaka City University Strategic Research Grant 2017 for basic researches, THE AMADA FOUNDATION Grant for Laser Processing Grant Number AF-2017224, and JSPS KAKENHI Grant Number JP26107002 in Scientific Research on Innovative Areas "Photosynergetics" to T. Y. T. O. thanks JSPS KAKENHI Grant Number $18 J 15442$ for JSPS Research Fellow. This work was performed under the Research Program for Next Generation Young Scientists of "Five-star Alliance" in "NJRC Mater. \& Dev" for T. O. and the Cooperative Research Program in "NJRC Mater. \& Dev." for T. Y. We thank Mr. Yuhei Tahara and Prof. Makoto Miyata for their help with TEM experiments, Mr. Yuichiro Hayasaka for his help with EDS measurements, and Mr. Shun. Ito for his help with HR-TEM and SAED measurements. We thank Mr. Kazuhiko Kondo of Thales Japan Inc. for his kind contribution to our laser system.

Keywords: bare particles $\bullet$ ionization $\bullet$ iron oxide $\cdot$ nanosecond laser $\cdot$ size distribution

[1] A.-H. Lu, E. L. Salabas, F. Schüth, Angew. Chem. Int. Ed. 2007, 46, 1222-1244.

[2] T. K. Jain, M. K. Reddy, M. A. Morales, D. L. Leslie-Pelecky, V. Labhasetwar, Mol. Pharm. 2008, 5, 316-327.

[3] T. Hyeon, Chem. Commun. 2003, 927-934.

[4] H. Xia, J. Wang, Y. Tian, Q.-D. Chen, X.-B. Du, Y.-L. Zhang, Y. He, H.B. Sun, Adv. Mater. 2010, 22, 3204-3207.

[5] J. B. Haun, T.-J. Yoon, H. Lee, R. Weissleder, Wiley Interdiscip. Rev. Nanomed. Nanobiotechnol. 2010, 2, 291-304.

[6] J. Gao, H. Gu, B. Xu, Acc. Chem. Res. 2009, 42, 1097-1107.

[7] T. K. Jain, M. A. Morales, S. K. Sahoo, D. L. Leslie-Pelecky, V. Labhasetwar, Mol. Pharm. 2005, 2, 194-205.

[8] H. M. T. Galvis, J. H. Bitter, C. B. Khare, M. Ruitenbeek, A. I. Dugulan, K. P. de Jong, Science 2012, 335, 835-838.

[9] A. Kay, I. Cesar, M. Grätzel, J. Am. Chem. Soc. 2006, 128, 15714 15721.

[10] Y. Hu, J. O. Jensen, W. Zhang, L. N. Cleemann, W. Xing, N. J. Bjerrum, Q. Li, Angew. Chem. Int. Ed. 2014, 53, 3675-3679.

[11] K.-C.Huang, S. H. Ehrman, Langmuir 2007, 23, 1419-1426.

[12] Y. Lu, Z. Zhu, Z. Liu, Carbon 2005, 43, 369-374.

[13] F. J. Douglas, D. A. MacLaren, M. Murrie, RSC Adv. 2012, 2, 80278035.

[14] K. Elihn, L. Landström, O. Alm, M. Boman, P. Heszler, J. Appl. Phys. 2007, 101, 034311.

[15] S. H. Huh, A. Nakajima, J. Appl. Phys. 2006, 99, 064302.

[16] Y. Hayasaki, T. Fukuda, T. Hasumura, T. Maekawa, Adv. Nat. Sci.: Nanosci. Nanotechnol. 2012, 3, 035010.

[17] T. Hasumura, T. Fukuda, R. L. D. Whitby, O. Aschenbrenner, T. Maekawa, J. Nanopart. Res. 2010, 13, 53-58.

[18] D. Zhang, B. Gökce, S. Barcikowski, Chem. Rev. 2017, 117, 3990-4103

[19] F. Mafuné, J. Kohno, Y. Takeda, T. Kondow, J. Phys. Chem. B 2000, 104, 9111-9117.
[20] A. Fojtik, A. Henglein, Ber. Bunsen-Ges. Phys. Chem. 1993, 97, 252254.

[21] J. Neddersen, G. Chumanov, T. M. Cotton, Appl. Spectrosc. 1993, 47, 1959-1964.

[22] H. Wang, O. Odawara, H. Wada, Appl. Surf. Sci. 2017, 425, 689-695.

[23] H. Wang, O. Odawara, H. Wada, Sci. Rep. 2016, 6, 20507.

[24] D. K. Park, S. J. Lee, J. H. Lee, M. Y. Choi, S. W. Han, Chem. Phys. Lett. 2010, 484, 254-257.

[25] H. J. Jung, M. Y. Choi, J .Phys. Chem. C 2014, 118, 14647-14654.

[26] N. Nakashima, K. Yamanaka, M. Saeki, H. Ohba, S. Taniguchi, T. Yatsuhashi, J. Photochem. Photobiol. A, 2016, 319, 70-77.

[27] T. Nakamura, H. Magara, Y. Herbani, S. Sato, Appl. Phys. A 2011, 104, 1021-1024

[28] T. Nakamura, Y. Mochidzuki, S. Sato, J. Mater. Res. 2008, 23, 968-974.

[29] T. Nakamura, K. Takasaki, A. Ito, S. Sato, Appl. Surfs. Sci. 2009, 255, 9630-9633.

[30] M. S. I. Sarker, T. Nakamura, S. Sato, J. Nanopart. Res. 2015, 17, 259.

[31] T. Nakamura, Y. Herbani, S. Sato, J. Nanopart. Res. 2012, 14, 785.

[32] T. Nakamura, S. Sato, J. Nanosci. Nanotechnol. 2015, 15, 426-732.

[33] J. L. H. Chau, C-Y. Chen, C-C. Yang, Arab. J. Chem. 2017, 10, S1395S1401.

[34] N. Nakashima, K. Yamanaka, A. Itoh, T. Yatsuhashi, Chin. J. Phys. 2014, 52, 504-518.

[35] D. Nishida, M. Kusaba, T. Yatsuhashi, N. Nakashima, Chem. Phys. Lett. 2008, 465, 238-240.

[36] D Nishida, E. Yamade, M. Kusaba, T. Yatsuhashi, N. Nakashima, J. Phys. Chem. A 2010, 1114, 5648-5654.

[37] A. Hu, J. Sanderson, Y. Zhou, W. W. Dulay, Diam. Relat. Mater. 2009 $18,999-1001$

[38] V. Amendola, P. Riello, M. Meneghetti, J. Phys. Chem. C 2011, 115 5140-5146.

[39] A. Kanitz, J. S. Hoppius, M. del Mar Sanz, M. Maicas, A. Ostendorf, E. L. Gurevich, ChemPhysChem 2017, 18, 1155-1164.

[40] A. De Bonis, T. Lovaglio, A. Galasso, A. Santagata, R. Teghil, Appl. Surf. Sci. 2015, 353, 433-438.

[41] B. K. Pandey, A. K. Shahi, J. Shah, R. K. Kotnala, R. Gopal, Appl. Surf. Sci. 2014, 289, 462-471.

[42] P. Liu, W. Cai, H. Zeng, J. Phys. Chem. C 2008, 112, 3261-3266.

[43] M. Ullmann, S. K. Friedlander, A. Schmidt-Ott, J. Nanopart. Res. 2002, 4, 499-509.

[44] J. B. Park, S. H. Jeong, M. S. Jeong, J. Y. Kim, B. K. Cho, Carbon 2008, 46, $1369-1377$.

[45] A. Ouchi, T. Tsunoda, Z. Bastl. M. Maryško. V. Vorliček. J. Boháček. K. Vacek. J. Pola, J. Photochem. Photobiol. A 2005, 171, 251-256.

[46] M. J. Wesolowski, S. Kuzmin, B. Wales, J. H. Sanderson, W. W. Duley, J. Mater. Sci. 2013, 48, 6212-6217.

[47] M. E. Fleet, Acta Crystallogr. B 1981, 37, 917-920.

[48] S. Sasaki, K. Nakamura, Y. Hamabe, E. Kurahashi. T. Hiroi, Nature 2001, 410, 555-557.

[49] T. Hamaguchi, T. Okamoto, K. Mitamura, K. Matsukawa, T. Yatsuhashi, Bull. Chem. Soc. Jpn. 2015, 88, 251-261.

[50] T. Yatsuhashi, N. Uchida, K. Nishikawa, Chem. Lett. 2012, 41, 722-724.

[51] T. Okamoto, K. Mitamura, T, Hamaguchi, K. Matsukawa, T. Yatsuhashi, ChemPhysChem 2017, 18, 1007-1011.

[52] T. Nakamura, Y. Mochidzuki, S. Sato, OSA Technical Digest on Conference on Lasers and Electro-Optics/Quantum Electronics and Laser Science Conference and Photonic Applications Systems Technologies, Baltimore, May, 2007, JThD89.

[53] Y. Sato, T. Kodama, H. Shiromaru, J. H. Sanderson, T. Fujino, Y. Eada, T. Wakabayashi, Y. Achiba, Carbon 2010, 48, 1673-1676. 
[54] S. L. Chin, S. Lagacé, Appl. Opt. 1996, 35, 907-911.

[55] S. Link, C. Burda, M. B. Mohamed, B. Nikoobakht, M. A. El-Sayed, J. Phys. Chem. A 1999, 103, 1165-1170.

[56] Y. Ishikawa, N. Koshizaki, A. Pyatenko, N. Saitoh, N. Yoshizawa, Y. Shimizu, J. Phys. Chem. C, 2016, 120, 2439-2446.

[57] Y. Ishikawa, N. Koshizaki, A. Pyatenko, Electron. Commun. Jpn. 2016, 99, 37-42.

[58] P. V. Kamat, M. Flumiani, G. V. Hartland, J. Phys. Chem. B 1998, 102, 3123-3128.

[59] L. Delfour, T.E. Itina, J. Phys. Chem. C 2015, 119, 13893-13900.

[60] D. Werner, A. Furube, T. Okamoto, S. Hashimoto, J. Phys. Chem. C 2011, 115, 8503-8512.

[61] F. Giammanco, E. Giorgetti, P. Marsili, A. Giusti, J. Phys. Chem. C 2010, 114, 3354-3363.

[62] K. Yamada, Y. Tokumoto, T. Nagata, F. Mafuné, J. Phys. Chem. B 2006, 110, 11751-11756.
[63] T. Yatsuhashi, N. Nakashima, J. Photochem. Photobiol. C 2018, 34, 52 84.

[64] N. Nakashima, S. Shimizu, T. Yatsuhashi, S. Sakabe, Y. Izawa, J. Photochem. Photobiol. C 2000, 1, 131-143.

[65] B. Shepperson, A. S. Chatterley, A. A. Sondergaard, L. Christiansen, M. Lemeshko, H. Stapelfeldt, J. Chem. Phys. 2017, 147, 013946.

[66] P.E. Mason, F. Uhlig, V. Vanek, T. Buttersack, S. Bauerecker, P. Jungwirth, Nat. Chem. 2015, 7, 250-254.

[67] S. L. Chin, S. A. Hosseini, W. Liu, Q. Luo, F. Theberge, N. Akozbek, A. Becker, V. P. Kandidov, O. G. Kosareva, H. Schröder, Can. J. Phys. 2005, 83, 863-905.

[68] T. Okamoto, E. Miyasaka, K. Mltamura, K. Matsukawa, T. Yatsuhashi, J. Photochem. Photobiol. A 2017, 344, 178-183.

[69] M. J. S. Monte, L. M. N. B. F. Santos, M. Fulem, J. M. S. Fonseca, C. A. D. Sousa, J. Chem. Eng. Data 2006, 51, 757-766. 
Entry for the Table of Contents (Please choose one layout)

Layout 1:

\section{ARTICLE}

Take off ligands and free from carbon shells: The use of aliphatic instead of aromatic solvent enable us to prepare bare, spherical, and constant size iron oxide nanoparticles from ferrocene even by prolonged femtosecond NIR laser irradiation. The liberated cyclopentadienyl ligands do not build carbon-shells but carbon agglomerates loosely surrounding nanoparticles (see picture).

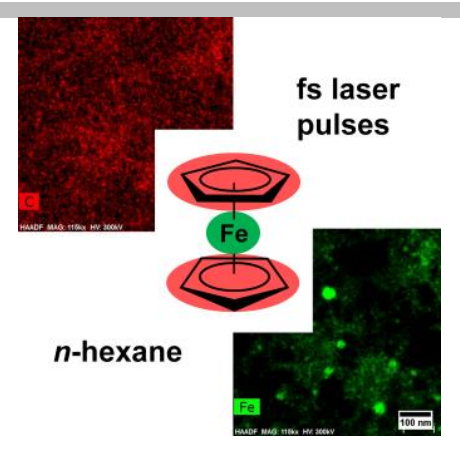

Takuya Okamoto, Takahiro Nakamura, Ryo Kihara, Tsuyoshi Asahi, Kenji Sakota, Tomoyuki Yatsuhashi*

Page No. - Page No.

Synthesis of Bare Iron Nanoparticles from Ferrocene Hexane Solution by Femtosecond Laser Pulses 